PROCEEDINGS OF THE

AMERICAN MATHEMATICAL SOCIETY

Volume 129, Number 7, Pages 2141-2156

S 0002-9939(01)05823-3

Article electronically published on February 23, 2001

\title{
KNOTS OF GENUS ONE OR ON THE NUMBER OF ALTERNATING KNOTS OF GIVEN GENUS
}

\author{
A. STOIMENOW
}

(Communicated by Ronald A. Fintushel)

\begin{abstract}
We prove that any non-hyperbolic genus one knot except the trefoil does not have a minimal canonical Seifert surface and that there are only polynomially many in the crossing number positive knots of given genus or given unknotting number.
\end{abstract}

\section{INTRODUCTION}

The motivation for the present paper came out of considerations of Gauß diagrams recently introduced by Polyak and Viro [23] and Fiedler [12] and their applications to positive knots [29].

For the definition of a positive crossing, positive knot, Gauß diagram, linked pair $p, q$ of crossings (denoted by $p \cap q$ ) see [29].

Among others, the Polyak-Viro-Fiedler formulas gave a new elegant proof that any positive diagram of the unknot has only reducible crossings. A "classical" argument rewritten in terms of Gauß diagrams is as follows: Let $D$ be such a diagram. Then the Seifert algorithm must give a disc on $D$ (see [9, 29]). Hence $n(D)=c(D)+1$, where $c(D)$ is the number of crossings of $D$ and $n(D)$ the number of its Seifert circles. Therefore, smoothing out each crossing in $D$ must augment the number of components. If there were a linked pair in $D$ (that is, a pair of crossings, such that smoothing them both out according to the usual skein rule, we obtain again a knot rather than a three component link diagram) we could choose it to be smoothed out at the beginning (since the result of smoothing out all crossings in $D$ obviously is independent of the order of smoothings) and smoothing out the second crossing in the linked pair would reduce the number of components. Hence $D$ has no linked pair, and so all crossings in $D$ are reducible.

\section{Knot DiAgrams With CANONICAL SEIFERT SURFACES OF GENUS ONE}

The starting point of our discussion here is in how far does the picture change when we consider $n(D)=c(D)-1$, that is, exactly one smoothing out of a crossing

Received by the editors February 11, 1999 and, in revised form, July 23, 1999 and October 20, 1999.

2000 Mathematics Subject Classification. Primary 57M27.

Key words and phrases. Positive knots, Seifert surfaces, Gauß diagrams, genus, unknotting number, alternating knots, Vassiliev invariants.

The author was supported by a DFG postdoc grant.

(C)2001 American Mathematical Society 
reduces the number of components and the genus of the canonical Seifert surface is one. Before we formulate the answer, let us make some definitions which will be of major importance in the following.

Definition 2.1. A collection of chords $a_{1}, \ldots, a_{n}$ in a Gauß diagram is called neighbored, if no two of them intersect and any other chord intersects either all or none of them.

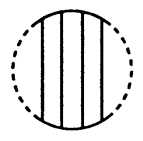

Any collection of neighbored chords has two outer chords (i.e., ones with respect to which the rest of the collection lies on one side) and the segments of the solid line belonging to the collection are the segments between the basepoints of the two outer chords containing basepoints of chords in the collection only.

We need some standard definitions, which we formulate here in our favorable Gauß diagram setting.

Definition 2.2. A knot diagram is reduced or irreducible if its Gauß diagram has no isolated chord; otherwise it is called reducible. A crossing corresponding to an isolated chord in a reducible knot diagram is called reducible. A diagram is called connected if the intersection graph of its Gauß diagram is so.

Definition 2.3. A knot diagram is twist reduced (or $\bar{t}_{2}$ irreducible) if it is reduced and there is no sequence of flypes

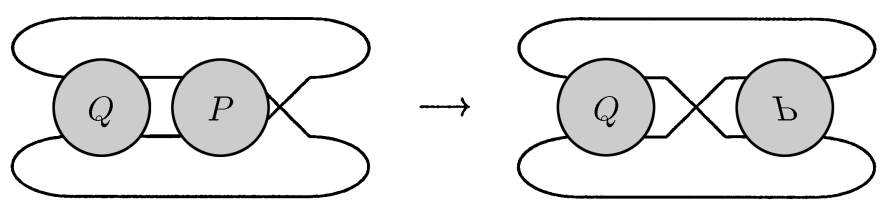

transforming it into a diagram on which a reducing $\bar{t}_{2}$ move

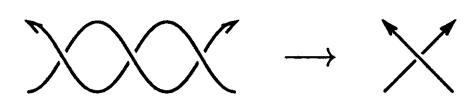

can be applied (the mirrored picture we understand as well as such a move). The reverse of this move we will just call a $\bar{t}_{2}$ move.

The $\bar{t}_{2}$ twist sequence or series of a diagram we will call the series of diagrams obtainable by applying $\bar{t}_{2}$ moves at $D$ 's crossings. Such a sequence can be parametrized by a tuple of integers corresponding to $D$ 's crossings (in some fixed order) indicating how many $\bar{t}_{2}$ moves are applied at any crossing.

The genus of a diagram we will call the genus of the surface, obtained by applying the Seifert algorithm to this diagram. Such a surface we will call canonical for the diagram.

Theorem 2.1. Let $D$ be a reduced diagram on which the Seifert algorithm gives a surface of genus one. Then $D$ is a rational knot diagram corresponding to the Conway notation [16] $C(p, q)$ with $p, q \in \mathbb{Z}$ non-zero and even, or a $(p, q, r)$-pretzel knot diagram $P(p, q, r)$ with $p, q, r$ odd. That is, genus one diagrams consist of the series of the (standard) trefoil and figure eight knot diagrams. 
This fact was also observed by Lee Rudolph [26, p. 4 top] without proof. Here we write one. Our approach will turn out more useful later, when we consider higher genera.

Proof. Let $D$ be such a diagram. First, $D$ is connected. Then $D$ has the following property: whenever a linked pair of crossings is smoothed out in $D$, the resulting diagram has only reducible crossings (that is, no linked pairs). Now in the smoothed out version the segments are run through in the following order:

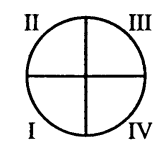

One convinces oneself that then in $D$ subdiagrams of the following kind do not occur:

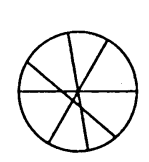

(a)

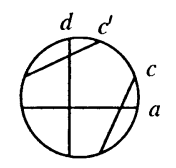

(b)

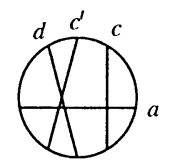

(c)

To do so, choose one linked pair, remove it, swapping 2 of the opposite segments into which the chords of the linked pair separate the solid line and observe that the two remaining chords are linked.

Now fix some chord $d$ in $D$. The exclusion of (b) and (c) shows that the following holds:

$$
a \cap c, a \cap d \Rightarrow c \cap d \vee \forall c^{\prime} \cap d: c \cap c^{\prime}
$$

In other words this means: for each two non-intersecting chords $c$ and $d$ intersecting a chord $a$, it holds that any other chord $c^{\prime}$ intersects either both or none of them.

Now make the following procedure: color $d$ blue and all $a$ with $a \cap d$ red. Assume now that any blue colored chord is intersecting any red colored chord and that some chord is not yet colored. Then (by connectedness of $D$ ) take one (call it $b$ ) intersecting some (w.l.o.g.) blue colored chord $c$. As $c$ intersects some (in fact, any) red colored chord $e$, either (i) $b$ intersects all chords intersecting $e$, in particular all blue chords, and then color $b$ red; or (ii) $b \cap e$. If $b$ intersects all colored chords intersecting $c$, then color $b$ blue. Else $b$ does not intersect some colored chord $f$ with $f \cap c$.

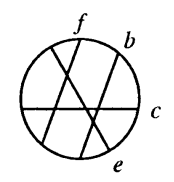

Then $f \cap e$ (else (c)) and so $\forall f^{\prime}: f^{\prime} \cap f \Rightarrow f^{\prime} \cap b$. Then give $b$ the color of $f$. Then one more chord is colored and still any blue colored chord intersects any red 
colored chord. At the end this procedure partitions all chords of $D$ into red and blue and $D$ looks like this:

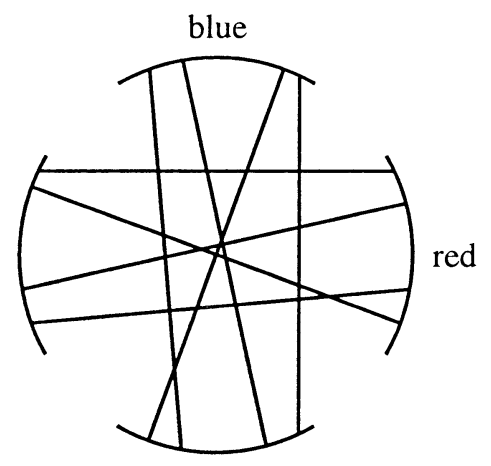

Now look at each partition separately. By exclusion of (a) and (b) and the fact that each blue chord is intersected by some fixed red chord, whenever two blue chords intersect, any other blue chord intersects exactly one of them. Then the partition looks like:

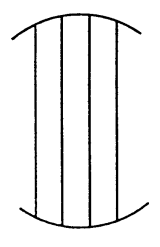

I

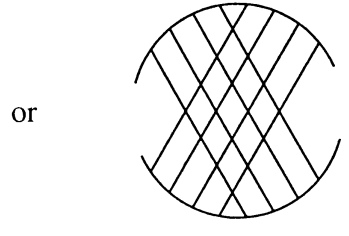

II

To see this, (re)color the blue chords black and white such that any chord intersecting a black (white) chord is colored white (black). Then by the above properties two chords intersect exactly if they have different color.

Now assume both partitions are of type I. Then by the even valence principle (each chord intersects an even number of chords) both have even number $p, q$ of chords and $D$ is the diagram $C(p, q)$ with $p, q$ even. Not both partitions can be of type II because of (a). Hence the remaining case is one partition of type I and one of type II. Then $D$ looks like:

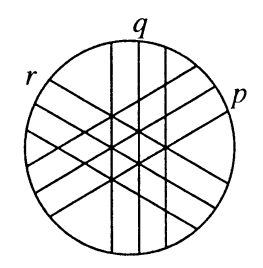

By even valence $p+q, p+r$ and $q+r$ must be even, so either $p, q, r$ even or $p, q, r$ odd. It is easily seen that for $p, q, r$ even $D$ is not realizable (or it can be formally checked using the conditions in [10]) hence $p, q, r$ are odd and $D$ is the pretzel diagram $P(p, q, r)$.

This theorem now has some consequences. Now, any $(p, q, r)$-pretzel knot is alternating for $p, q, r>0$ or by [1, exercise 5.32, p. 149] alternating or almost 
alternating for $p, q>0, r<0$. This covers all cases up to obversion (that is, taking the mirror image), so we obtain

Corollary 2.1. Any genus one knot, that is neither alternating nor almost alternating, has no minimal canonical Seifert surface.

Using that the only genus one torus knot is the trefoil and that any nonhyperbolic knot is composite (so of genus at least two), satellite (so by [19] nonalternating and by [2] non-almost alternating) or a torus knot, we obtain the statement of the abstract.

Corollary 2.2. Any non-hyperbolic genus one knot except the trefoil does not have a minimal canonical Seifert surface.

A series of such knots are Whitehead doubles of (non-trivial) knots, and so we see that any Whitehead double of a (non-trivial) knot $K$ does not have a minimal canonical Seifert surface. This was known for lonely $K$ (that is, $K$ is not a satellite itself) by [36] (see the proof of theorem 7 of [9]). Morton 21] gave an independent argument for showing the non-existence of minimal canonical Seifert surfaces using the HOMFLY polynomial and giving the example of a Whitehead double of the trefoil.

Corollary 2.3. Let $K$ be an alternating genus one knot. Then $K=P(p, q, r)$ with $p, q, r>0$ odd, or $K=C(p, q)$ with $p, q>0$ even (or their obverse).

Proof. Take an alternating diagram of $K$. Then the Seifert algorithm must give a genus one surface on it [13] 9] and now apply Theorem 2.1 .

Corollary 2.4. Let $K$ be an positive genus one knot. Then $K=P(p, q, r)$ with $p, q, r>0$ odd.

Proof. Take a positive diagram of $K$ and again use the minimality of the canonical Seifert surface. Then by Theorem $2.1 D$ is either $C(p,-q)$ with $p, q>0$ even or $P(p, q, r)$ with $p, q, r>0$ odd. However, the former case in contained in the latter, as $C(p,-q)=P(p-1,1, q-1)$ (see the continued fractions [1] $\S 2.3]$ ).

Now we come to our result on unknotting numbers.

Corollary 2.5. Let $K$ be a positive unknotting number one knot. Then $K$ is a twist knot (i.e., a rational knot with Conway notation $C(2, n)$ or $C(n, 2), n \in \mathbb{N})$.

Proof. Let $K$ be such a knot. Then by Corollary 1 of [26] a positive unknotting number one knot has also genus one and then by Corollary [2.4 $K$ is a $(p, q, r)$ pretzel knot with $p, q, r$ odd. Now, by Corollary 2 of [17], a $(p, q, r)$-pretzel knot with $p, q, r$ odd has unknotting number 1 if and only if $\{1,1\},\{-1,-1\},\{-3,1\}$, or $\{-1,3\} \subset\{p, q, r\}$, in which case the knot is a twist knot.

Remark 2.1. Only odd crossing number twist knots occur. It can be shown that even crossing number twist knots are not positive. For example, thay have negative Casson invariant (which can be most easily seen from its Polyak-Viro formula [23]) and negative minimal degree of the Jones polynomial (it is -2) contradicting the obstructions to positivity of [9] and [31]. Moreover, even crossing number twist knots have zero signature, contradicting the property of [8, corollary 3.4, p. 497].

Remark 2.2. J. Przytycki informed me of an early draft by himself and Taniyama [25], where he obtained the same and some similar results. 
Corollary 2.6. Any positive genus one knot has at most two positive (unoriented) diagrams (in $S^{2}$ ), at most one of the kind $C(p,-q), p, q>0$ even, and at most one of the kind $P(p, q, r), p, q, r>0$ odd.

Proof. It follows from Theorem 2.1 that all diagrams $C(p,-q)$ depict distinct rational knots, as the expression $C\left(a_{1}, \ldots, a_{n}\right)$ with all $a_{i}$ even is unique for any rational knot. In the pretzel case note that the diagrams $P(p, q, r)$ with $p, q, r>0$ odd are alternating and do not admit a flype 20 (modulo permuting $p, q$ and $r$, which however is also realized by orientation reversal and isotopies of the diagram in $S^{2}$ ).

Example 2.1. $C(4,-4)$ and $P(3,1,3)$ are the only positive diagrams of the knot 74 .

Example 2.2. Any $(p, q, r)$-pretzel knot with $p, q, r>1$ odd is not rational (e.g., again by the above flyping argument, as rational knots are alternating) and hence its $(p, q, r)$-pretzel diagram is its unique positive diagram.

Conjecture 2.1. These are the only positive knots with only one positive diagram.

Theorem 2.1 allows us to reproduce in a much easier way [32, corollary 3.1]:

Corollary 2.7. Any connected almost positive unknot diagram is a one crossing diagram or an unknotted twist knot diagram.

Proof. By the inequality of of Bennequin-Vogel (see [29]) the canonical Seifert surface of such a diagram must have genus at most one. The genus zero case is the one crossing diagram, so look at the genus one case. Switching a crossing in a positive canonical genus one diagram and possibly annihilating it by a Reidemeister II move with another one gives a diagram of (i) $C(p,-q)$ with $p, q \geq 0$ even and not $p=q=0$ or (ii) $P(p, q, r)$ with $p, q, r$ odd, $q, r>0$ and $p \geq-1$. Case (i) with $p q=0$ comes (before the Reidemeister move) from a diagram of the desired type, and for $p q>0$ the diagram is knotted (e.g., as positive, connected and of more than one crossing). A direct calculation of $v_{3}$ of 29] shows that case (ii) is a knotted diagram unless $p=-1 \wedge(q=1 \vee r=1)$ as desired.

Finally, we repeat some results of Rudolph [26] slightly simplifying their proofs by not involving counts of Seifert circles (his notations $O_{\geq}, O_{<}$).

Theorem 2.2. Let $D$ be a k-almost positive diagram of an achiral or slice knot. Then the canonical Seifert surface of such a diagram has genus at most $k$.

Sketch of the proof. For slice use the Kronheimer-Mrowka-Menasco-Bennequin inequality with the slice genus instead of the Seifert genus (called in [26] "slice Bennequin inequality (sBi)"). For achiral knots reduce it to the slice case by Lee Rudolph's trick (consider $D \# D$ ).

Corollary 2.8. Any positive non-trivial knot is non-slice.

Proof. See the arguments in the introduction and combine them with Theorem 2.2 . 
Corollary 2.9. Any non-trivial positive and almost positive knot is chiral and nonslice.

Proof. Let $K$ be an achiral or slice non-trivial positive or almost positive knot. Then using Theorem 2.2 and the arguments in the proof of Corollary 2.7 it has either a connected positive diagram of $>1$ crossing, contradicting Corollary 2.8 or a diagram $P(p,-1, q)=C(p,-1, q)$ with $p, q>1$ odd. But such a knot is rational and has a positive rational diagram; hence it is again by Corollary 2.8 chiral and non-slice, contradicting the assumption.

Corollaries 2.8 and 2.9 are obtained also in $8,25,34$, always using the signature.

\section{HIGHER GENERA}

Theorem 3.1. There exist numbers $c_{n}, n \in \mathbb{N}$, with $c_{n}=O\left(8^{n}\right)$ such that any twist reduced diagram of Seifert genus $n$ has at most $c_{n}$ crossings. Therefore, any $k n o t$ diagram of Seifert genus $n$ lies in the $\bar{t}_{2}$ twist sequence of some diagram of at most $c_{n}$ crossings.

To explain in the following what happens we need some definitions. We consider first the flypes in more detail.

Definition 3.1. The flype in (1) is called the flype at crossing $p$, where $p$ is the distinguished crossing in both diagrams not belonging to the tangles $P$ and $Q$ (and their transforms). If a diagram admits a flype at crossing $p$, then we say that $p$ admits a flype.

There is an evident bijection between the crossings of the diagram before and after the flype, so that we can we can trace a crossing in a sequence of flypes and identify it with its image in the transformed diagram when convenient.

When considering orientation, according to the orientation near $p$ we distinguish two versions of flypes we call types $\mathrm{A}$ and $\mathrm{B}$, whose difference will be very important in the following. The diagram on the right hand-side of (1) for these two versions of the flype is given on Figure 1 Note that any crossing admits a flype of at most one type.

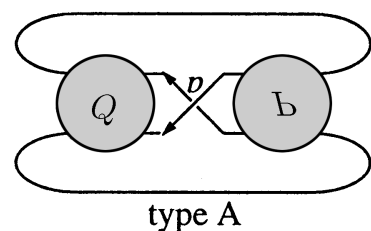

Figure 1. A flype of type A and B

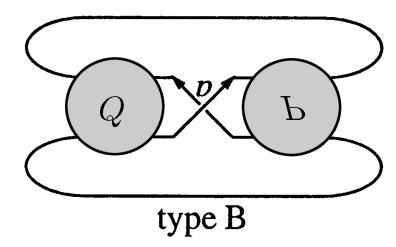

Definition 3.2. We call two crossings $p$ and $q$ of a knot diagram linked, notationally $p \cap q$, if the crossing strands are passed in cyclic order $p q p q$ along the solid line, and unlinked if the cyclic order is ppqq. We call two crossings $p$ and $q$ equivalent if they are linked with the same set of other chords, that is if $\forall c \neq a, b: c \cap a \Longleftrightarrow c \cap b$. We call $p$ and $q \sim$-equivalent $p \sim q$ if they are

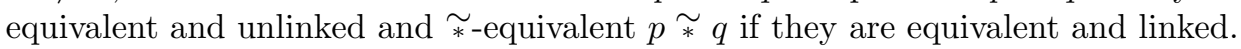


It is an exercise to check that $\sim$-equivalence and $\approx$-equivalence are indeed equivalence relations and that two crossings are $\sim$ - (resp. $\approx-$ ) equivalent if and only if after a sequence of type B (resp. type A) flypes they can be made to form a reverse (resp. parallel) clasp.

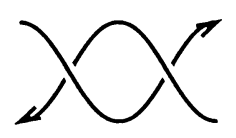

reverse clasp

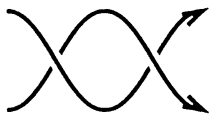

parallel clasp

Proof of Theorem 3.1. We proved that we can set $c_{1}:=4$. Now we use induction and consider a twist reduced diagram $D$ of Seifert genus $n+1$. Fix a linked pair $(p, q)$ in $D$. Build $D^{\prime}$ and $D^{\prime \prime}$ as follows:
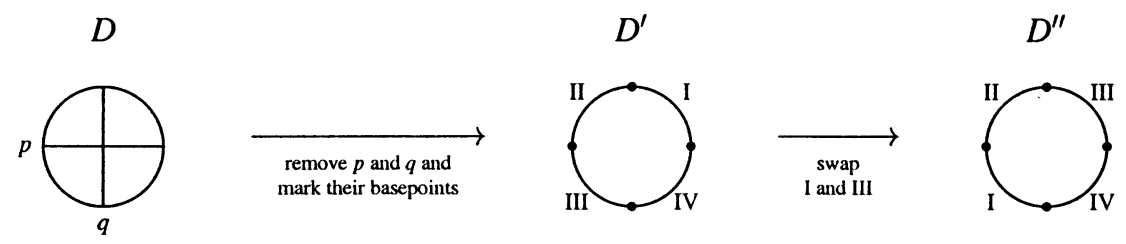

Then $D$ and $D^{\prime \prime}$ are realizable (i.e., correspond to a knot diagram), but $D^{\prime}$ may not be so. Moreover, $D^{\prime \prime}$ is of Seifert genus $n$.

Assume $D^{\prime \prime}$ has reducible chords. Now, one can see that the linking status of two chords in $D^{\prime \prime}$ is different from this in $D$ iff (a) one of the two chords is linked with both $p$ and $q$ and the other one is linked with either $p$ or $q$ in $D$ or (b) the one chord is linked with $p$, but not $q$, and the other chord is linked with $q$, but not $p$. Hence there are three kinds of reducible chords in $D^{\prime \prime}$ :

1) linked exactly with both $p$ and $q$ and with exactly those chords linked with either $p$ or $q$ in $D$,

2) linked with $p$, but not $q$, and exactly those chords linked with $q$ in $D$,

3) linked with $q$, but not $p$, and exactly those chords linked with $p$ in $D$.

If one of the first group has more than 2 elements or one of the other two has more than one element, then by type B flypes looking on Gauß diagrams like

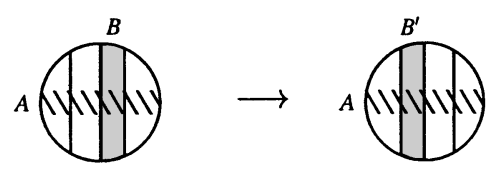

you can get these $\geq 3$ chords (resp. 2 chords together with $p$ or $q$ ) neighbored and $D$ becomes reducible. Hence $D^{\prime \prime}$ has at most 4 reducible chords.

Now assume, removing these reducible chords from $D^{\prime \prime}$ and calling the result $D^{\prime \prime \prime}, D^{\prime \prime \prime}$ has more than $8 c_{n}$ chords. Then by induction it, and also $D^{\prime \prime}$, has at least $9 \sim$-equivalent chords obtained by $\bar{t}_{2}$ twists and possible subsequent flypes. Now, putting the 4 basepoints of $p$ and $q$ on the solid line of $D^{\prime \prime}$ will still leave a collection of $3 \sim$-equivalent chords $a, b$, and $c$ in $D^{\prime \prime}$ that have in $D$ the same linking status with $p$ and $q$. But then by the above remarks they are linked with the same sets of 
(other) chords in $D$ as well, because the linking status in $D$ is determined by the linking status in $D^{\prime \prime}$ and the position w.r.t. the basepoints of $p$ and $q$ in $D$, which for all of $a, b$ and $c$ is the same. Hence this collection will remain $\sim$-equivalent by exchanging segments I and III and reinstalling $p$ and $q$, and $\sim$-equivalent chords can be made to form a reverse clasp by flypes, so $D$ is twist reducible after possible flypes, a contradiction.

Hence $D^{\prime \prime \prime}$ has at most $8 c_{n}$ chords, $D^{\prime \prime}$ has at most $8 c_{n}+4$ chords and $D$ has at most $c_{n+1} \leq 8 c_{n}+6$ chords.

The proof together with $c_{1}=4$ shows that we have $c_{n} \leq \frac{34 \cdot 8^{n-1}-6}{7}$. This bound is clearly quite unsharp and very likely it can be improved.

Remark 3.1. The description of how the linking status changes by smoothing a linked pair and the fact that a diagram is of genus 0 iff it has no linked pair inductively show that the genus of a diagram depends only on the intersection graph of its Gauß diagram. A special case of a conjecture of Chmutov and Duzhin [7, §4.2.3] (slightly reformulated for realizable Gauß diagrams and without considering relations) asserts that this intersection graph determines the diagram up to (iterated) mutations, so our observation of invariance of the diagram genus can be considered as some small evidence of this conjecture.

Corollary 3.1. As a function of their crossing number $c(K)$, there are only polynomially many alternating knots $K$ of given genus $g(K)$ and positive knots of given genus or given unknotting number $u(K)$. That is,

$$
\#\{K: K \text { positive knot, } c(K)=n, g(K)=g\}=O_{n}\left(n^{p_{g}}\right)
$$

(and the same for $u$ resp. alternating knots) for some number $p_{g} \in \mathbb{N}$, where $O_{n}$ denotes the asymptotic behaviour as $n \rightarrow \infty$.

Proof. The statements for the genus of an alternating knot follow from the fact that alternating and positive knots have minimal (genus) canonical Seifert surfaces and that flypes preserve the knot type.

For $K$ alternating use that an alternating diagram of $K$ has minimal crossing number by [15, 22, 33. For $K$ positive use that if $K$ has a positive diagram of $c$ crossings, then by [29, theorem 6.1] and [24, theorem 2.2.E], $c(K) \geq \sqrt{2 c}$.

For the statement about the unknotting number, use the inequality in corollary 1 of [26] implying that the genus of a positive knot is at most equal to its unknotting number (see also [29]).

The proof of the Tait flyping conjecture 20] allows a more specific statement about the alternating case. It is most elegantly expressed using the following:

Definition 3.3. A sequence $\left\{a_{n}\right\}_{n=1}^{\infty}$ is called periodically polynomial in $n$ with period $d \in \mathbb{N}$ if there are polynomials $P_{0}, \ldots, P_{d-1} \in \mathbb{Q}[n]$, such that $a_{n}=P_{n \bmod d}(n)$, and periodically polynomial if it is periodically polynomial with period $d$ for some $d \in \mathbb{N}$.

Corollary 3.2. The number of alternating knots of genus $g$ and $n$ crossings (as well as the number of such knots up to in- and/or obversion and the number of invertible and/or achiral ones among them) is periodically polynomial in $n$ for almost all $n$ (that is, with finitely many exceptional values of $n$ ). 
This fact follows from standard combinatorial arguments, but maybe it is desirable to explain them in more detail.

Sketch of the proof. Parametrize as above a $\bar{t}_{2}$ twist sequence of diagrams of genus $g$ by the number of twists at each crossing of the corresponding $\bar{t}_{2}$ irreducible diagram $D$.

We start by the observation that if a crossing $p$ of a non-trivial (that is, of more than one element) -equivalence class does not admit a type A flype, and if it admits a type B flype, then it does so after any number of $\bar{t}_{2}$ twists at it.

Now, for any $\bar{t}_{2}$ irreducible diagram $D$ of genus $g$ let $l=l_{D}$ be the number of trivial $\sim$-equivalence classes of its crossings. Then the braiding sequence generated by $D$ we decompose into $2^{l}$ subsequences, sorting a diagram $D^{\prime}$ in $D^{\prime}$ s sequence according to whether or not each of the $l \sim$-equivalence classes in $D^{\prime}$ is trivial or not.

The previous observation now says that if a diagram in one of these subsequences $B$ occurs in another subsequence $B^{\prime}$, then $B$ and $B^{\prime}$ contain diagrams of the same knots. Therefore, we can discard all duplicated subsequences and are left with caring about duplications in any subsequence itself.

Thus, we can enumerate the number of knots in each $\bar{t}_{2}$ twist subsequence separately. Now, by flyping the diagram so -equivalent chords become neighbored, any such subsequence can be represented by a Gauß diagram with thickened chords, depicting non-trivial collections of neighbored chords.

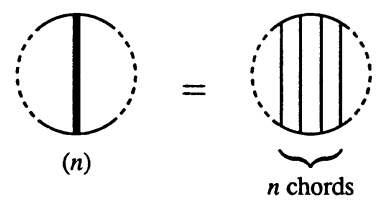

Ordering in a fixed manner these thickened chords, flype transformations of the diagram descend to permutations of the thickened chords therein. Then we count composition 11 of the crossing number of fixed length modulo the action of a subgroup of the symmetric group. That is, if the $c$ crossings decompose into $l$ trivial, $m$ non-trivial odd size and $n$ even size $\sim$-equivalence classes, we consider

$$
\begin{aligned}
\mathcal{M}:=\left\{\left(a_{1}, \ldots, a_{m}, b_{1}, \ldots, b_{n}\right)\right. & \in \mathbb{N}^{m+n}: \\
\sum a_{i}+\sum b_{j} & \left.=c-l, a_{i} \geq 3 \text { odd, } b_{i} \text { even }\right\} / G,
\end{aligned}
$$

with $G$ being a subgroup of $S_{m} \times S_{n}$. Allowing in- and obversion, the picture remains the same, just the group $G$ generally becomes larger.

By Burnside's lemma [14, lemma 14.3 on p. 1058], $|\mathcal{M}|$ is the arithmetic mean of the cardinalities of the fix point sets of the action of each element of $G$, which are tuples as in (2) with additional conditions of the kind $a_{i}=a_{j}$ with $1 \leq i<j \leq m$ and/or $b_{i}=b_{j}$ with $1 \leq i<j \leq n$ for specific pairs $(i, j)$. That is, this reduces to enumerating compositions with specified parts equal. But such counts admit straightforward recursive formulae, from which their periodical polynomiality is evident.

Arguing this way for each series separately gives the assertion.

\footnotetext{
${ }^{1}$ Representations of a natural number as the sum of natural numbers whose order is relevant and not necessarily de- or increasing.
} 
The origin of the exceptional cases in the beginning is the fact that each sequence starts giving a contribution only from a certain crossing number on. This way we see that the number of exceptions may a priori be larger than $c_{g}$, but it certainly will not be larger than

$$
\max _{D} c(D)+2 c^{\prime}(D),
$$

where $D$ runs over all $\bar{t}_{2}$ irreducible diagrams of genus $g$ and $c^{\prime}(D)$ the number of crossings of $D$ admitting a type A flype (that is, a flype which is destroyed by a $\bar{t}_{2}$ move near this crossing).

Remark 3.2. The proof suggests that the period will generally not be much smaller than the least common multiple of the lengths of the orbits of the crossings in the $\bar{t}_{2}$ irreducible diagrams of genus $g$ under their symmetries, and as the number of diagrams is already expected to grow rapidly with $g$, so will the number of symmetries, and hence the period. Therefore, the phenomenon of the above corollary will be hardly empirically visible even for small $g$.

Alternatively, a periodically polynomial sequence $\left(a_{n}\right)$ can be defined by the property, that its generating function

$$
f(x)=\sum_{i=0}^{\infty} a_{i} x^{i}
$$

is a rational function $f(x)=\frac{P(x)}{Q(x)}$, with $P, Q \in \mathbb{Z}[x]$ and the zeros of $Q$ being all (complex) roots of unity. The degree of $\left(a_{n}\right)$, that is, the maximal degree of all $P_{i}$ in Definition 3.3 is then one less than the highest multiplicity of such a root of unity as zero of $Q$, and the period of $\left(a_{n}\right)$ is the least common order of all such roots. Although we cannot give any nice property of the period, as for the degree $d_{g}$, we note that Corollary 3.2 implies the following identity, where $\mathcal{A}_{n, g}$ is the set of alternating genus $g$ knots of $n$ crossings:

$$
d_{g}=\min \left\{i \in \mathbb{N}: \limsup _{n \rightarrow \infty} \frac{\left|\mathcal{A}_{n, g}\right|}{n^{i}}=0\right\} .
$$

This identity can be taken as a self-contained definition of $d_{g}$, but it reveals little about its nature. Thus we can set $p_{g}=d_{g}$ in Corollary 3.1 for alternating knots and $p_{g}=2 d_{g}$ for positive knots.

Remark 3.3. It is also worth mentioning that by the same (slice) Bennequin inequality arguments as in Corollary 1 of [26] Corollary 3.1 extends (for both genus and unknotting number) to $k$-almost positive knots for any given $k$, if we replace the crossing number of the knot by the minimal crossing number of a $k$-almost positive diagram of it (for the definition and properties of $k$-almost positive knots, I propose to the reader to consult [25, 31]). Unfortunately, yet, besides the positive case ([29, cor. 6.2]), there is no inequality relation (in the non-trivial direction) available between both crossing numbers. If such inequality exists, already considering Whitehead doubles (whose number grows exponentially in the crossing number; see remarks after Question 5.1), one sees that generically a knot is not $k$-almost positive for any finite choice of values of $k$, and hence in particular $k$-almost positive knots exist for infinitely many $k$. This fact was established in [31] using the Jones polynomial. Note, contrarily, that it is not yet clear whether for all, almost all or 
for which $k$ there are $k$-almost positive knots. Although both arguments, the one here and that of [31], are in principle constructive, they will be very hard to apply for high values of $k$, unless more structure and regularity is exhibited to avoid the resulting calculational explosion.

Let $d(K)$ denote the bridge number of $K$ and $\tilde{g}(K)$ the weak genus of $K$, i.e., the minimal genus of all its diagrams.

Our next corollary addresses Vassiliev invariants, and more precisely the question: how well are Vassiliev invariants determined by their values on knots of given genus? Clearly there are Vassiliev invariants vanishing on knots of bounded genus the Conway (polynomial) Vassiliev invariants. But already asking for primitive Vassiliev invariants with this property seems non-trivial (see Conjecture 5.1). Here we obtain the answer for the weak genus.

Corollary 3.3. Fix $g \in \mathbb{N}$. Then for $n \rightarrow \infty$ there are more than polynomially many (in n) linearly independent primitive Vassiliev invariants of degree $n$ vanishing on knots of weak genus at most $g$.

Proof. By Corollary 3.1 and the braiding polynomial arguments of [28] the space of Vassiliev invariants of degree $n$ restricted to knots of weak genus at most $g$ is polynomially bounded (above) in $n$. Then the assertion follows from the result of [6].

Contrast this with

Theorem 3.2 (see [30]). Let $S \subset \mathbb{N}_{+}$be infinite. Then if some Vassiliev invariant $v$ vanishes on all alternating knots $K$ with $g(K)=\tilde{g}(K) \in S$, then $v \equiv 0$.

Contrary to this we ask

Question 3.1. Is a Vassiliev invariant $v$ of degree at most $n$, such that $v(K)=0$ if $K$ has a canonical Seifert surface of genus at most $n$, zero?

The reason for this question is the desire to prove an exponential upper bound in $n$ for the number of Vassiliev invariants of degree at most $n$, which would follow from [28, if the answer to Question 3.1] is positive.

Compare this with Conjecture 5.1 and the following two theorems.

Theorem 3.3 (see [27, 28]). Any Vassiliev invariant vanishing on alternating knots is zero.

Theorem 3.4 (see [28]). Any Vassiliev invariant vanishing on positive knots is zero.

Then we have

Corollary 3.4. The bridge number $d(K)$ is bounded for all knots $K$ by a function of $\tilde{g}(K)$. This means there is a function $f: \mathbb{N} \rightarrow \mathbb{N}$ such that $d(K) \leq f(\tilde{g}(K))$ for any knot $K$.

Proof. This is clear from the previous remarks, as you can isotope in the plane any diagram near a crossing so as $\bar{t}_{2}$ moves at that crossing not to create additional local extrema.

Question 3.2. Is there a function $f_{0}: \mathbb{N} \rightarrow \mathbb{N}$ such that $d(K) \leq f_{0}(g(K))$ for any knot $K$ ? 
Remark 3.4. Promising candidates for a sequence of knots of fixed $g$ but unbounded $d$ are easily found, for example Whitehead doubles of knots of unboundedly growing bridge number, so the main difficulty in answering this question seems the lack of nice lower bounds for $d(K)$.

The last consequence generalizes a result of [29] and answers a question posed therein.

Corollary 3.5. There are only finitely many alternating (in fact, homogeneous [9]) knots with the same Alexander polynomial.

Proof. We use the terminology of [9, §1]. Assume there is an infinite family of such knots. Then they all have the same weak genus [9, corollary 4.1] and hence by Theorem 3.1 there is an infinite family $\mathcal{F}$ of such knots in the same $\bar{t}_{2}$ twist sequence of some diagram $D$. A $\bar{t}_{2}$ twist corresponds to inserting a vertex $p$ on an edge $e$ of the Seifert graph of $D$. W.l.o.g. $e$ is not an isthmus (else $D$ is composite and the $\bar{t}_{2}$ twist corresponds to twisting one of the components, which preserves the knot type); hence $p$ is not a cut vertex and so lies on some circle in its block of the Seifert graph. Therefore, $\mathcal{F}$ contains diagrams with the maximal circle length of the Seifert graph blocks arbitrarily large, which by the proof of theorem 5 of 9] renders the top degree coefficient of $\Delta$ arbitrarily large, contradicting the assumption.

\section{A REMARK ON LINKS}

So far we developed the theory only for knots. It is worth saying some words on links.

It turns out that the picture is basically the same here. Let $g(L)$ be the genus of $L$ and $c(L)$ the number of components of $L$. Call $\hat{g}(L)=g(L)+c(L)-1$ the modified genus of $L$ (for knots $g=\hat{g}$ ). Having a non-split link diagram, by replacing crossings of different components by parallel clasps (which preserves the modified genus), we get back to the knot case. In fact we see that the numbers $d_{g}^{c}$ (defined analogously to $d_{g}$, but for $c$ component links of modified genus $g$ ) satisfy $d_{g}^{c} \leq d_{g}-c+1$, and so, for example, you can show that the sequences

$$
\frac{\sum_{c>1 \text { odd }} a_{n, g}^{c}}{a_{n, g}}, \quad \frac{\sum_{c \text { even }} a_{n, g}^{c}}{a_{n \pm 1, g}}, \quad \text { and therefore also } \frac{\sum_{c>1} a_{n, g}^{c}}{a_{n, g}+a_{n \pm 1, g}}
$$

for fixed $g$ tend to zero as $n \rightarrow \infty$, where

$$
a_{n, g}^{c}:=\#\{L \text { alternating non-split link of } c \text { components }
$$

$$
\text { with } \hat{g}(L)=g \text { and } c(L)=n\}
$$

and $a_{n, g}:=a_{n, g}^{1}$. That is, alternating knots present the richest variety on alternating links of given modified genus.

This property will extend to split links without trivial split components, if the following conjecture is true.

Conjecture 4.1. The $d$-numbers are strongly superadditive, that is, $d_{a+b}>d_{a}+d_{b}$ for any positive $a, b \in \mathbb{N}$. 


\section{Some QUestions AND CONJECTURES REvisited}

We review several questions and conjectures in the light of the preceding results. The first question is related to Vassiliev invariants [3, 5].

Conjecture 5.1. There is no primitive Vassiliev invariant vanishing on knots of genus one (or weaker, of bounded genus).

As we observed, the condition that the Vassiliev invariant be primitive (and the genus be bounded) is essential. Moreover, for a (potential) proof we will need the vanishing on (genus one) knots without minimal canonical Seifert surfaces. The case that Whitehead doubles as a special class of such knots has been considered independently before in different contexts, so it is worth establishing some connection.

It has been observed by McDaniel and Rong [18], that dualizing the (untwisted) Whitehead double operation (even with any tangle instead of a clasp) on the space of knot invariants induces an endomorphism of the filtered (but not graded) space $\mathcal{V}=$ $\bigcup_{n=1}^{\infty} \mathcal{V}^{n}$ of Vassiliev invariants (the filtration given by subspaces $\mathcal{V}^{n}$ of invariants of degree at most $n$ ). An invariant of the kind considered in the conjecture clearly lies in the kernel of this map. This is of course just a necessary condition, and the primitive Vassiliev invariant of degree 2 (killed by this map) is easily observed not to have the property in the conjecture. Anyway, the conjecture inspires the quest for some understanding of this kernel. However, the dimension of this kernel on $\mathcal{V}^{n} / \mathcal{V}^{n-1}$ is quite large - it is at least $1 /(n-1)$ of the whole dimension of $\mathcal{V}^{n} / \mathcal{V}^{n-1}$. In fact, this map is nilpotent on each $\mathcal{V}^{n}$, because of the observation of Lin (see [4] p. 283, (ii)]) that ( $n$-fold) iterated Whitehead doubles are $n$-trivial. So there may be very many primitive Vassiliev invariants lying in the kernel. On the other hand, it is not clear how large the space of primitive invariants is. We do not yet know for example whether

$$
\frac{\operatorname{dim}\{\text { primitive Vassiliev invariants of degree } n\}}{\operatorname{dim}\{\text { all Vassiliev invariants of degree } n\}} \stackrel{n \rightarrow \infty}{\rightarrow} 0 .
$$

So there are many things that can happen ...

Another question was posed by C. Adams [1]. Roughly it is:

Question 5.1. How many knots possess minimal canonical Seifert surfaces?

Using Theorem 2.1 we can answer the question for genus one knots: up to a given crossing number polynomially many out of exponentially many. (We will in the following observe that the results of $\$ 3$ suggest a similar(ly bad) situation for any fixed genus.) That there are exponentially many genus one knots for bounded crossing number follows from the fact that the Whitehead doubles of distinct knots are distinct, their crossing number is linearly bounded in the crossing number of their companion and that the number of knots of given crossing number has an exponential lower bound [11. (On the other hand, there are at most exponentially many knots of fixed crossing number at all [35.) Therefore, there are exponentially many Whitehead doubles of crossing number at most $n$ and so also exponentially many of crossing number exactly $n$ for infinitely many $n$ (as the partial sum sequence of some sequence $\left(a_{n}\right)$ grows weaker than any exponential $C^{n}$ in $n, C>1$, if $\left(a_{n}\right)$ does so).

Question 5.2 (see [29]). Does any positive knot realize its unknotting number in a positive diagram? 
Corollary 2.5 allows us to answer positively this question for unknotting number one. (In fact, it was my result of [32], that connected almost positive unknot diagrams are unknotted twist knot diagrams, which inspired me to prove corollary [2.5). The general answer appears by far not trivial, though.

Question 5.3. Which alternating knots have unknotting number one? Does any alternating unknotting number one knot realize its unknotting number in an alternating diagram?

Concerning the first part of the question, there are alternating non-twist knots of unknotting number one. $8_{14}$ and $7_{6}$ are examples. (An unknotted version of $7_{6}$ can be found in [1] fig. 5.59, p. 151].) Note, that, unlike $8_{14}$ and $7_{6}$, in general an alternating knot does not realize its unknotting number in an alternating diagram. The first such example, $10_{8}$, is due to Bleiler and Nakanishi [1, p. 73] and has unknotting number two. Therefore, the second part of the question. Note also, that as a consequence of the Tait flyping conjecture [20, each alternating diagram of an alternating knot has the same unknotting number, so each alternating knot realizes its unknotting number either in any or in no alternating diagram (see [29. remark 3.7]).

\section{ACKNOWLEDGEMENT}

I would wish to thank to the referee, S. Bleiler, S. Boyer, D. Lines, T. Kobayashi, J. Przytycki, T. Cochran, R. Fintushel, and T. Kanenobu for helpful remarks and discussions and to L. Paris, B. Perron and O. Couture for their help at Dijon.

\section{REFERENCES}

1. C. C. Adams, Das Knotenbuch, Spektrum Akademischer Verlag, Berlin, 1995 (The knot book, W. H. Freeman \& Co., New York, 1994). MR 94m:57007

2. — et al., Almost alternating links, Topol. Appl. 46 (1992), 151-165. MR 93h:57005

3. D. Bar-Natan, On the Vassiliev knot invariants, Topology 34 (1995) 423-472. MR 97d:57004

4. J. S. Birman, New points of view in knot theory, Bull. Amer. Math. Soc. 28 (1993), 253-287. MR 94b:57007

5. J. S. Birman and X-S. Lin, Knot polynomials and Vassiliev's invariants, Invent. Math. 111 (1993) 225-270. MR 94d:57010

6. S. V. Chmutov and S. V. Duzhin, A lower bound for the number of Vassiliev knot invariants, Topology Appl. 92 (1999), 201-223. MR 2000b:57016

7. _ Kontsevich integral, Preprint of Max-Planck-Institut für Mathematik (Bonn, August 1997); available via anonymous file transfer from pier.botik.ru, subdirectory pub/local/zmr, file name ki.ps.gz.

8. T. D. Cochran and Robert E. Gompf, Applications of Donaldson's theorems to classical knot concordance, homology 3-spheres and Property P, Topology 27(4) (1988), 495-512. MR 90g:57020

9. P. R. Cromwell, Homogeneous links, J. London Math. Soc. (series 2$) 39$ (1989), 535-552. MR 90f:57001

10. C. H. Dowker and M. B. Thistlethwaite, Classification of knot projections, Topol. Appl. 16 (1983), 19-31. MR 85e:57003

11. C. Ernst and D. W. Sumners, The Growth of the Number of Prime Knots, Proc. Cambridge Phil. Soc. 102 (1987), 303-315. MR 88m:57006

12. T. Fiedler, Gauss sum invariants for knots and links, UPS Toulouse monography, to appear.

13. D. Gabai, Genera of the alternating links, Duke Math. J. 53(3) (1986), 677-681.

14. Handbook of Combinatorics, Vol. II (P. L. Graham, M. Grötschel and L. Lovácz, eds.), North-Holland, 1995. MR 96h:05001 
15. L. H. Kauffman, New invariants in the theory of knots, Amer. Math. Mon. 95 (1988), 195-242. MR 89d:57005

16. T. Kanenobu, Examples on polynomial invariants for knots and links, Math. Ann. $\mathbf{2 7 5}$ (1986), 555-572. MR 88b:57010

17. T. Kobayashi, Minimal genus Seifert surfaces for unknotting number 1 knots, Kobe J. Math. 6 (1989), 53-62. MR 90k:57008

18. M. McDaniel and Y. Rong, On the dimensions of Vassiliev invariants coming from link polynomials, George Washington University preprint, March 1997.

19. W. W. Menasco, Closed incompressible surfaces in alternating knot and link complements, Topology 23 (1) (1986), 37-44. MR 86b:57004

20. W. W. Menasco and M. B. Thistlethwaite, The Tait flyping conjecture, Bull. Amer. Math. Soc. 25 (2) (1991), 403-412. MR 92b:57017

21. H. R. Morton, Seifert circles and knot polynomials, Proc. Camb. Phil. Soc. 99 (1986), 107-109. MR 87c:57006

22. K. Murasugi, Jones polynomial and classical conjectures in knot theory, Topology 26 (1987), 187-194. MR 88m:57010

23. M. Polyak and O. Viro, Gauss diagram formulas for Vassiliev invariants, Int. Math. Res. Notes 11 (1994) 445-454. MR 95k:57012

24.

25. J. H. Przytycki and K. Taniyama, Almost positive links have negative signature, draft, 1991.

26. L. Rudolph, Positive links are strongly quasipositive, preprint, available at the math preprint server, preprint number 9804003.

27. T. Stanford, Braid commutators and Vassiliev invariants, Pacific Jour. of Math. 174 (1) (1996). MR 97i:57008

28. A. Stoimenow, Gauß sum invariants, Vassiliev invariants and braiding sequences, J. of Knot Theory and Its Ram. 9 (2) (2000), 221-269.

29. _ Positive knots, closed braids and the Jones polynomial, preprint.

30. Genera of knots and Vassiliev invariants, Jour. of Knot Theory and its Ramifications 8(2) (1999), 253-259. MR 2000b:57019

31. _ On some restrictions to the values of the Jones polynomial, preprint

32. Gauss sums on almost positive knots, preprint.

33. M. B. Thistlethwaite, A spanning tree expansion for the Jones polynomial, Topology 26 (1987) 297-309. MR 88h:57007

34. P. Traczyk, Non-trivial negative links have positive signature, Manuscripta Math. 61 (1988), 279-284. MR 89g:57010

35. D. J. A. Welsh, On the number of knots and links, Sets, graphs and numbers (Budapest, 1991), 713-718, Colloq. Math. Soc. János Bolyai, 60, North-Holland, Amsterdam, 1992. MR 94f: 57010

36. W. C. Whitten, Isotopy types of minimal knot spanning surfaces, Topology 12 (1973), 373-380. MR 51:9046

Ludwig-Maximilians University Munich, Mathematics Institute, Theresienstrasse 39, 80333 MünChen, Germany

E-mail address: stoimeno@informatik.hu-berlin.de

URL: http://www.informatik.hu-berlin.de/〜stoimeno

Current address: Max Planck Institute of Mathematics, P.O. Box 7280, D-53072 Bonn, Germany

E-mail address: alex@mpim-bonn.mpg.de 